\title{
Calculating Singlet Excited States: Comparison with Fast Time- Resolved Infrared Spectroscopy of Coumarins
}

\author{
Magnus W. D. Hanson-Heine ${ }^{\dagger}$ Alisdair Wriglesworth ${ }^{\dagger}{ }^{\text {Maliha Uroos }},{ }^{\dagger}$ James A. Calladine, ${ }^{\dagger}$ \\ Thomas S. Murphy, ${ }^{\dagger}$ Michelle Hamilton, ${ }^{\dagger}$ Ian P. Clark, ${ }^{\ddagger}$ Michael Towrie,${ }^{\dagger}$ James Dowden, ${ }^{\dagger}$ \\ Nicholas A. Besley, ${ }^{\dagger, *}$ and Michael W. George ${ }^{\dagger, \pi, *}$ \\ ${ }^{\dagger}$ School of Chemistry, University of Nottingham, University Park, Nottingham, NG7 2RD, UK. \\ "Department of Chemical and Environmental Engineering, University of Nottingham Ningbo China, \\ 199 Talking East Road, Ningbo 315100, China. \\ ${ }^{\ddagger}$ Central Laser Facility, Science \& Technology Facilities Council, Research Complex at Harwell, \\ Rutherford Appleton Laboratory, Didcot, OXI 1 0QX, UK.
}

Email:mike.george@nottingham.ac.uk

Email:nick.besley@nottingham.ac.uk

\begin{abstract}
In contrast to the ground state, the calculation of the infrared (IR) spectroscopy of molecular singlet excited states represents a substantial challenge. Here we use the structural IR fingerprint of the singlet excited states of a range of coumarin dyes to assess the accuracy of density functional theory based methods for the calculation of excited state IR spectroscopy. It is shown that excited state Kohn-Sham density functional theory provides a high level of accuracy and represents an alternative approach to time-dependent density functional theory for simulating the IR spectroscopy of the singlet excited states.
\end{abstract}

\section{Introduction}

Computational chemistry has impact right across molecular science. While computational methods for determining the structure and properties of molecules in their electronic ground state are well established to the extent that such calculations are often considered as standard practice $^{1-5}$ a different picture emerges if one considers calculations for molecules in electronically excited states, particularly singlet excited states. Accurate and computationally 
expedient methods to calculate excited states would benefit a wide range of research applications, including modeling excited state dynamics and the photochemical activation of molecules and materials.

Various computational approaches have been utilized for calculating excited states, including complete active space self-consistent field coupled with multiconfigurational perturbation theory (CASPT) ${ }^{6}$ multi-reference configuration interaction (MRCI) ${ }^{7}$ coupled cluster methods, ${ }^{8,} 9$ and propagator approaches. However, these techniques quickly become prohibitively expensive as the size of the system increases restricting their use for larger systems. In addition such methods often require an expert user, inhibiting their use by the wider community.

In contrast, adiabatic linear-response time-dependent density functional theory (TDDFT) ${ }^{10,11}$ is relatively straightforward to use and provides a good balance between calculation speed and accuracy and is increasingly used for calculating a wide range of excited state properties for progressively larger molecules. ${ }^{12-19}$ Despite its growing popularity, TDDFT has many associated problems and has been shown to be inaccurate for long-range charge transfer, ${ }^{20,21}$ Rydberg, ${ }^{22,23}$ and core excitations, ${ }^{24}$ when using common forms for the exchange-correlation functional. This has led to the development of range-separated functionals which can rectify many of these problems, albeit with an increase in computational cost. ${ }^{24-26}$ However, in some cases these functionals show larger errors than conventional functionals, ${ }^{27}$ especially for calculating accurate vibrational frequencies, ${ }^{28}$ and there is evidence to suggest that TDDFT predicts the potential energy surface less accurately compared to ground state DFT methods. $^{29,30}$ 
An alternative approach to such problems is to apply ground state methods directly to study excited states. While this is straightforward for the lowest energy state of a given multiplicity, for example the lowest triplet excited state $\left(T_{1}\right)$ of a molecule with a singlet ground state, in order to compute other excited states such as the $\mathrm{S}_{1}$ state, some modifications to the methodology are required to prevent variational collapse to the electronic ground state during the self-consistent field (SCF) process. This has led to the development of excited state DFT techniques (eDFT). ${ }^{31-37}$ In this study we use the maximum overlap method (MOM) ${ }^{35}$ developed by Gill and co-workers in which a non-Aufbau occupancy is maintained during the SCF orbital optimization procedure, without adding significant computational cost compared to an analogous ground state calculation. A recent study that considered the vibrational frequencies of singlet excited states of small molecules demonstrated that more accurate frequencies were obtained using eDFT with the MOM procedure compared to TDDFT, ${ }^{30}$ and showed that accurate prediction of the vibrational frequencies does not necessarily correlate with accurate prediction of the excitation energy. The comparison of experimental and predicted vibrational frequencies of the singlet excited states reveals how accurately the shape of the potential energy surface is described by the calculations in the vicinity of a geometry minimum. The study of excited state IR spectroscopy of larger systems is also frustrated by the lack of suitable experimental data.

Here, we present the predicted infrared spectra of the $S_{1}$ excited states of a range of coumarin dyes obtained by fast time-resolved infrared spectroscopy (TRIR) ${ }^{38,}, 39$ and assess the accuracy of computed spectra from TDDFT and eDFT methodologies. These studies demonstrate excellent agreement between experimental and predicted frequencies arising from eDFT calculations at a computational cost sufficiently low to be of potentially wide utility. 


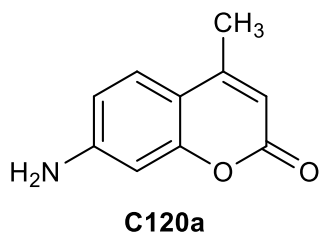<smiles>Cc1cc(=O)oc2cc(N)ccc12</smiles><smiles>Nc1ccc2c(C(F)(F)F)cc(=O)oc2c1</smiles><smiles>Cc1cc(=O)oc2c3c4c(cc12)CCCN4CCC3</smiles><smiles>O=c1cc(C(F)(F)F)c2cc3c4c(c2o1)CCCN4CCC3</smiles>

Figure 1. Molecular structures of the 7-aminocoumarins used in this study.

The 7-aminocoumarins that have been used in this study (Figure 1) were selected as they have a number of properties that make for a good theoretical benchmark for TRIR spectroscopy on molecules of this size. 7-aminocoumarins are commonly used as laser dyes due to their high quantum yields of emission and low lying $\pi \pi^{*}$ excited states of essentially HOMO to LUMO character. ${ }^{40-45}$ The first excited singlet states of the 7 -aminocoumarins exhibit relatively strong solvatochromic behavior, which has been widely attributed to partial intramolecular charge transfer character (ICT) between the nitrogen atom and carbonyl oxygen atom following extensive studies using TDDFT, ${ }^{41-45}$ together with emission and absorption spectroscopy. ${ }^{46-48}$ Despite this charge separation, unsubstituted 7-aminocoumarins are too small for the partial ICT character to be considered as a long-range charge transfer excited state in TDDFT, and B3LYP therefore performs adequately for calculating excitation energies. $^{41-45}$ This is important for comparing with TRIR data, as the B3LYP functional is also known to reproduce ground state experimental frequencies well using both scaled harmonic $^{49,50}$ and anharmonic ${ }^{2,51}$ techniques, while other commonly used functionals, such as $\mathrm{PBE}^{52}$ or $\mathrm{CAM}-\mathrm{B} 3 \mathrm{LYP}^{25}$ and other range corrected functionals often predict frequencies of significantly lower quality. ${ }^{50,53}$ 
Coumarins containing an unsubstituted 7- $\mathrm{NH}_{2}$ group also show higher fluorescent quantum yields in polar solvents than in non-polar solvents, ${ }^{54,55}$ attributed to an increase in positive charge on the $\mathrm{N}$-atom and simultaneous flattening of the $7-\mathrm{NH}_{2}$ group in polar solvents, leading to suppression of non-radiative decay from the excited state. ${ }^{56}$

Lower fluorescence quantum yields in highly polar solvents have been attributed to the formation of a separate, low-lying, twisted intramolecular charge transfer (TICT) $\mathrm{S}_{1}$ state in 7-aminocoumarins containing flexible alkyl-substituted $7-\mathrm{NH}_{2}$ groups. ${ }^{48,54-57}$ This has been supported by TDDFT calculations, ${ }^{58}$ and appears to require range-separated functionals for a correct description within TDDFT. ${ }^{58}$ Furthermore, the conformational flexibility of these systems may also influence the IR spectra. Consequently, 7-aminocoumarins substituted with flexible alkyl chains have been avoided in this work so as to provide a more accurate comparison between TDDFT and eDFT vibrational frequencies. Currently, it remains an open question to the importance of range-separated functionals for the prediction of the IR spectroscopy of these states since the shape of the excited state potential energy surface is most relevant and not the energy difference with the ground state surface.

\section{Experimental Details}

Coumarin 102, 120a and 153 (Exciton), coumarin 151 (Sigma Aldrich) along with deuterated acetonitrile (Sigma Aldrich) were all used as received. ${ }^{13} \mathrm{C}$ labelled coumarin $120 \mathrm{~b}$ was synthesised using an adapted literature procedure (see Supplementary Material). ${ }^{59}$ The concentrations of the samples were such that the absorbance at $266 \mathrm{~nm}$ was $c a .0 .5$. For all of the IR measurements, a transmission IR cell (Harrick Scientific Products, Inc.) with $\mathrm{CaF}_{2}$ windows $(25 \times 2 \mathrm{~mm})$ at a path length of $0.15 \mathrm{~mm}$ was used. 
FTIR spectra were measured on a Nicolet Avatar 380 spectrometer at $2 \mathrm{~cm}^{-1}$ resolution. The ultrafast TRIR experiments were conducted on the ULTRA system at the STFC Rutherford Appleton Laboratories, and the apparatus is discussed in detail elsewhere. ${ }^{60}$ Briefly, a cryogenically cooled Ti:sapphire laser amplifier (Thales Laser ALPHA 10000) produces 800 nm laser pulses $(0.8 \mathrm{~mJ}, 10 \mathrm{kHz}$ with a pulsewidth of $50 \mathrm{fs})$. The laser output is split, with one portion used to generate the $266 \mathrm{~nm}$ pump beam by harmonic generation, and the second to produce the probe. For the mid-IR probe, the $800 \mathrm{~nm}$ laser output is sent through an optical parametric amplifier (Light Conversion TOPAS) to produce signal and idler pulses. Difference frequency generation is then used on the signal and idler to produce the mid-IR probe.

After passing through the sample, the IR probe is dispersed onto two linear 128 element MCT detector arrays to acquire spectra at $10 \mathrm{kHz}$. By measuring pump ON and pump OFF, difference spectra are generated.

\section{Computational Details}

Ground and excited state DFT calculations were performed using the Q-Chem software package, ${ }^{61}$ with the B3LYP functional, ${ }^{62,}{ }^{63} 6-311 \mathrm{G}(\mathrm{d}, \mathrm{p})$ basis set, and standard SG-1 numerical integration grid. ${ }^{64}$ The calculated species were optimized to minimum energy geometries, followed by a normal mode harmonic frequency analysis using analytical second derivatives of the energy with respect to nuclear displacement. Harmonic frequencies were scaled using a scaling factor of $0.9682 .{ }^{2,50,65}$ In principle it would be possible to derive scaling factors for different excited state methods. However, there is currently a lack of experimental data encompassing a wide range of functional groups to make this reliable, and we assume here that scaling factors derived for the ground state can be applied with no 
additional modifications following previous excited state frequency benchmarking studies. ${ }^{30}$ Electronic absorption and emission energies were calculated by taking vertical $\mathrm{S}_{1} \leftarrow \mathrm{S}_{0}$ and $\mathrm{S}_{0} \leftarrow \mathrm{S}_{1}$ transitions using the ground state and $\mathrm{S}_{1}$ excited state optimized geometries, respectively. Open-shell singlet eDFT calculations were performed using unrestricted DFT with the maximum overlap method invoked to prevent the variational collapse to the ground state $^{35}$ The molecular orbitals of the ground state were used as the starting point for the eDFT calculations, with a $\beta$-electron moved from the HOMO to the LUMO to form the spinmixed state $\left(\mathrm{eDFT}^{\mathrm{SM}}\right)$. Subsequently, energies, analytic gradients, and Hessian matrices were modified according to the Ziegler post-SCF spin-purification formula of equation (1) to give the spin-purified state $\left(\mathrm{eDFT}^{\mathrm{SP}}\right)$ in order to compensate for the use of a single determinant wavefunction, ${ }^{66}$

$$
E=2 E_{S}-E_{T}
$$

where $E$ is the energy of the true singlet state, $E_{S}$ is the energy of the single determinant spinmixed state and $E_{T}$ is the energy of the corresponding triplet state. These values were calculated by combining energies, gradients and Hessian matrices obtained from separate SCF calculations on the singlet and triplet states. This methodology can be successfully applied for states that are dominated by a single excitation from the ground state wave function, which is the case for the states considered here.

\section{Results and Discussion}

Although the intention of this work is to examine the effectiveness of eDFT for predicting the vibrational fingerprint of coumarin excited states in order to assess its potential to provide a 
more general tool for predicting and assigning infrared spectra of excited states, the nature of the lowest lying singlet excited state, $\mathrm{S}_{1}$, will be discussed before detailed analysis of the infrared data. The highest occupied molecular orbitals (HOMOs) and lowest unoccupied molecular orbitals (LUMOs) (Figure 2) are delocalized over the molecules. All four HOMOs have a significant contribution from the benzene rings and the substituent nitrogen atoms, while the LUMOs are more localized on the cyclic-ester rings and oxygen atoms. This is consistent with an ICT model for the $\mathrm{S}_{1}$ excited state and high fluorescent quantum yields have been observed for all the coumarins in acetonitrile, ${ }^{46-48}$ indicating an ICT excited state in this polar solvent. ${ }^{46-48}$ The trifluoromethyl analogues, C151 and C153, show no significant changes in their HOMOs when compared with their non-fluorinated counterparts, while the LUMO densities are drawn away from the ester rings and onto the $\mathrm{CF}_{3}$ groups. For ringsubstitution at the 7-amino position, there is little change in the LUMOs of C102 and C153, while the HOMOs are extended onto the additional alkyl rings. 
(a)


(b)


(c)


Figure 2. HOMO and LUMO molecular orbital plots for: (a) C120, (b) C151, (c) C102, and (d) C153.

Calculated vertical excitation and emission energies between the $\mathrm{S}_{0}$ and $\mathrm{S}_{1}$ states conform with the previously reported values for TDDFT in the literature. ${ }^{43-45}$ The calculated absorption and emission vertical transitions are on average 18 and $26 \mathrm{~nm}$ lower than experimental absorption and emission maxima recorded in cyclohexane, respectively (Table 1 in the Supplementary Material). ${ }^{46-48,59}$ Uncorrected eDFT $^{\mathrm{SM}}$ vertical excitation energies significantly underestimate the true values for low-lying singlet states. ${ }^{30,35,67}$ The spin- 
purified $\mathrm{eDFT}^{\mathrm{SP}}$ vertical transitions are on average $5 \mathrm{~nm}$ and $14 \mathrm{~nm}$ larger than the experimental maxima for absorption and emission, respectively. The predicted geometries of the coumarins are comparable across all three excited state methods, and can be found in the Supplementary Material. ${ }^{59}$

(a)

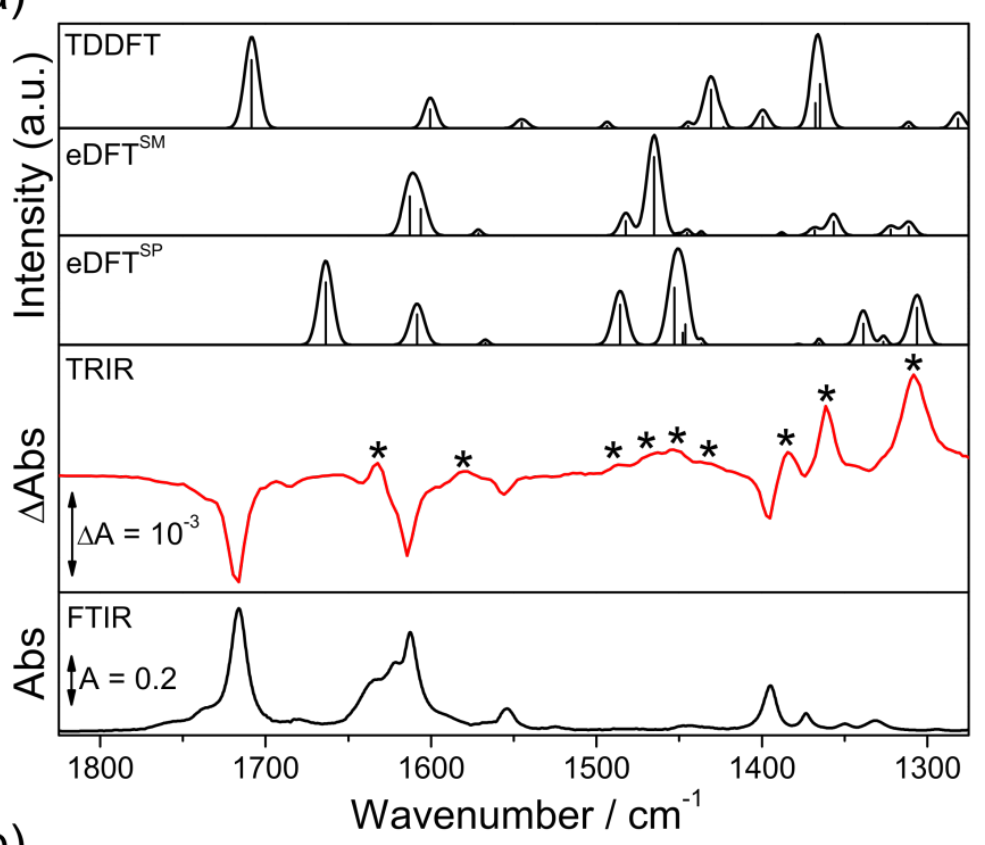

(b)




Figure 3. FTIR and TRIR spectra of (a) C120a and (b) $2,4-{ }^{13} \mathrm{C}_{2}$-labelled C120b obtained 100 ps after excitation at $266 \mathrm{~nm}$ in acetonitrile- $d_{3}$. The $\mathrm{S}_{1}$ frequency calculations (TDDFT, $\mathrm{eDFT}^{\mathrm{SM}}$ and $\mathrm{eDFT} \mathrm{SP}^{\mathrm{SP}}$ ) are shown for comparison. The peaks labelled * are assigned to the $\mathrm{S}_{1}$ excited state.

Figure 3 shows the ground state FTIR and the TRIR spectrum obtained 100 ps following excitation (266 nm) of (a) C120a and (b) $2,4-{ }^{13} \mathrm{C}_{2}$-labelled C120b in acetonitrile- $d_{3}$. It is clear that excitation leads to bleaching of the ground state and the production of positive bands which are assigned to the singlet excited state. For the purpose of assessing the quality of the calculations as a tool to aid experimental TRIR spectroscopic assignments, comparisons are initially drawn between the TRIR spectra and the theoretical predictions for C120a and $\mathrm{C} 120 \mathrm{~b}$ in the $\mathrm{S}_{1}$ state following excitation at $266 \mathrm{~nm}$. For $\mathrm{C} 120 \mathrm{a}$ the highest frequency vibrational band in the spectral region examined shifts from 1716 to $1632 \mathrm{~cm}^{-1}$ (Figure 3), consistent with the assignment of the $\mathrm{S}_{1}$ state as ${ }^{1} \pi \pi^{*}$ with a degree of ICT character. The intensity of this peak is partially masked in the TRIR spectrum by an overlapping ground state bleach. Another small peak is also observed at $1580 \mathrm{~cm}^{-1}$. An accurate description of the highest frequency band is particularly important since it contains a high degree of carbonyl stretching motion, and is therefore a useful band for probing the nature of organic excited states. Both the $\mathrm{eDFT}^{\mathrm{SM}}$ and $\mathrm{eDFT}^{\mathrm{SP}}$ calculations reproduce these two high frequency vibrational modes well, with a similar overestimation of the carbonyl band for $\operatorname{eDFT}^{\mathrm{SP}}(31$ $\left.\mathrm{cm}^{-1}\right)$ as observed for the ground state $\left(44 \mathrm{~cm}^{-1}\right)$. In contrast, TDDFT overestimates the carbonyl band by $76 \mathrm{~cm}^{-1}$, while the lower of the two modes is reproduced well. Calculations using an explicit solvent model consisting of a single acetonitrile- $d_{3}$ molecule positioned on the carbonyl group (see Table 1 and Figures 1 to 3 in the Supplementary Material) ${ }^{59}$ further reduced overestimation of the carbonyl mode frequency for all three calculations, bringing the $\mathrm{eDFT}^{\mathrm{SP}}$ result within $10 \mathrm{~cm}^{-1}$ of the experimental value. 
The TRIR spectra also show a cluster of peaks between 1500 and $1420 \mathrm{~cm}^{-1}$ related to the $\mathrm{S}_{1}$ state in addition to intense bands at 1384, 1361 and $1308 \mathrm{~cm}^{-1}$. Again, the two eDFT calculations provide a good match with the experiment, while TDDFT predicts the peaks at ca. $1450 \mathrm{~cm}^{-1}$ to be much more evenly spread out over the spectral region, rather than overlapping as observed in the experiment. The positions of the low frequency peaks appear to be better reproduced by eDFT, although the predicted intensity of the band at $1361 \mathrm{~cm}^{-1}$ from the TDDFT calculations correlates more with the experimental observation. Overall, both eDFT methods produce a more accurate $S_{1}$ spectrum than TDDFT for C120a. Spinpurification also offers a slight improvement over the $\mathrm{eDFT}^{\mathrm{SM}}$ spectrum. However, all three methods struggle to predict the correct intensities for the middle and lower frequency bands.

Normal mode analysis of C120a (Figure 4) shows the three highest frequency vibrational modes to be relatively localized, with the highest frequency mode containing largely carbonyl stretching character. The two modes at lower energies contain a mixture of carbon-nitrogen, and carbon-carbon stretching motion as well as a noticeable degree of $\mathrm{NH}_{2}$ bending character. TDDFT and $\mathrm{eDFT}^{\mathrm{SP}}$ predict similar atomic displacements, and while $\mathrm{eDFT}^{\mathrm{SM}}$ predicts significant delocalization of the carbonyl mode onto the aryl ring system and $\mathrm{NH}_{2}$ group. This discrepancy is potentially an artefact of the spin-mixed state. 


\section{TDDFT}
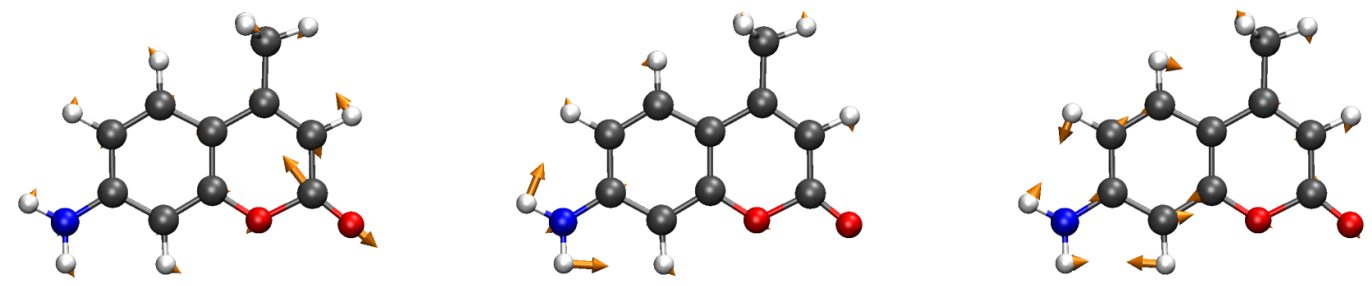

\section{eDFTSP}
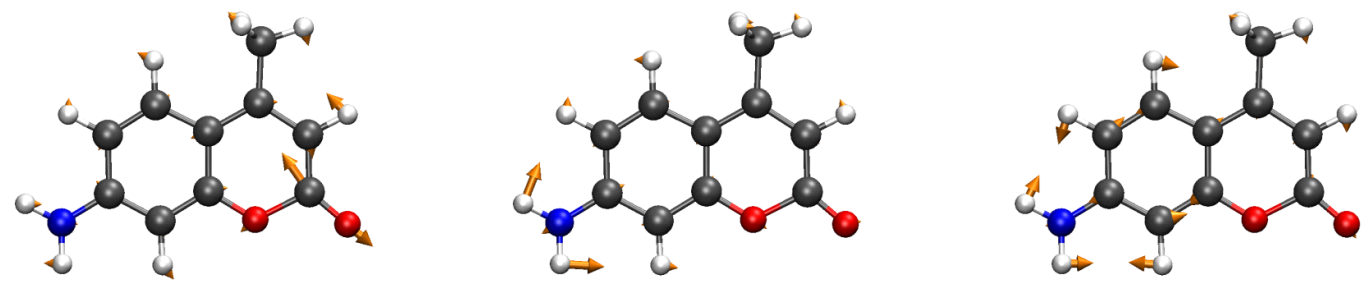

\section{eDFT $^{S M}$}
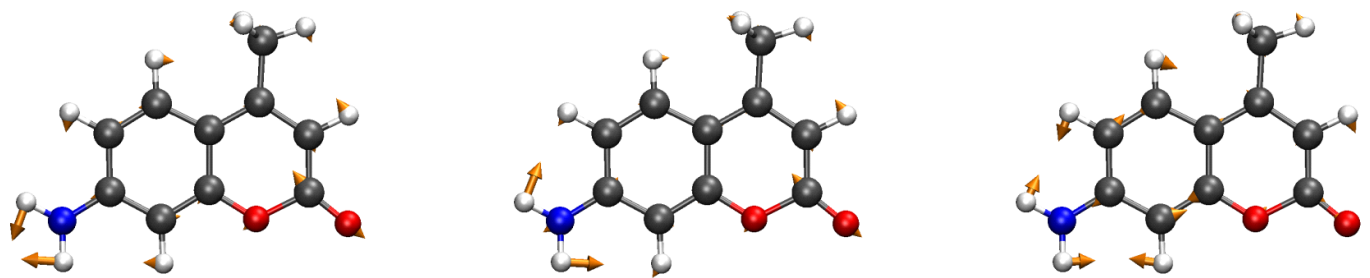

Figure 4. Atomic displacements for the three highest frequency normal modes of the ${ }^{1} \pi \pi^{*}$ state of C120a in the spectral region examined, shown in descending frequency order from left to right: at 1708,1600 , and $1545 \mathrm{~cm}^{-1}$ for TDDFT; 1664,1608 , and $1567 \mathrm{~cm}^{-1}$ for $\mathrm{eDFT}^{\mathrm{SP}}$; and at 1613,1606 , and $1571 \mathrm{~cm}^{-1}$ for $\mathrm{eDFT} \mathrm{SM}^{\mathrm{SM}}$.

In the case of $\mathrm{C} 120 \mathrm{~b}$ with ${ }^{13} \mathrm{C}$ at positions 2 and 4 (Figure 1), the ground state carbonyl peak downshifts from 1716 to $1684 \mathrm{~cm}^{-1}$, causing only minor changes to the rest of the spectrum compared to the non-labelled equivalent. The high frequency band in the $\mathrm{S}_{1}$ spectrum is also better resolved on downshifting from 1632 to $1591 \mathrm{~cm}^{-1}$. This confirms the eDFT ${ }^{\mathrm{SP}}$ and TDDFT normal mode assignments for the highest frequency band, as increasing the mass of the carbonyl group is expected to have a large effect on a mode containing a high degree of 
carbonyl stretching character. There are also two shoulders on the carbonyl peak of the excited state at $1579 \mathrm{~cm}^{-1}$ and $1563 \mathrm{~cm}^{-1}$. Both eDFT methods represent these three peaks reasonably well, whereas the gap between the three modes is too large with TDDFT. In the C120b TRIR spectrum the observation of the excited state peak at $c a .1308 \mathrm{~cm}^{-1}$ was not clear since it is near the edge of the acquisition window in this experiment. In general though, eDFT provides a good match for the middle and lower parts of the IR spectrum, as expected from the ${ }^{12} \mathrm{C}$ calculations. 
(a)

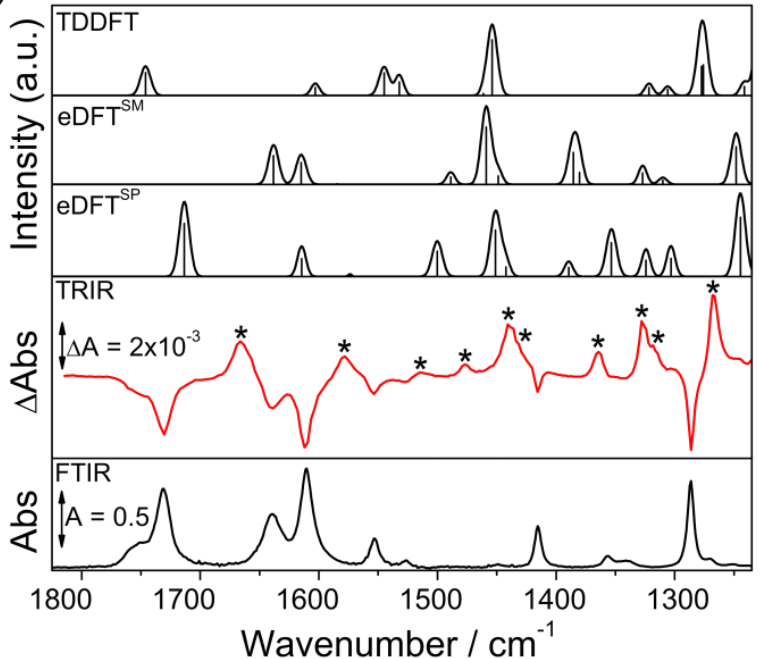

(b)

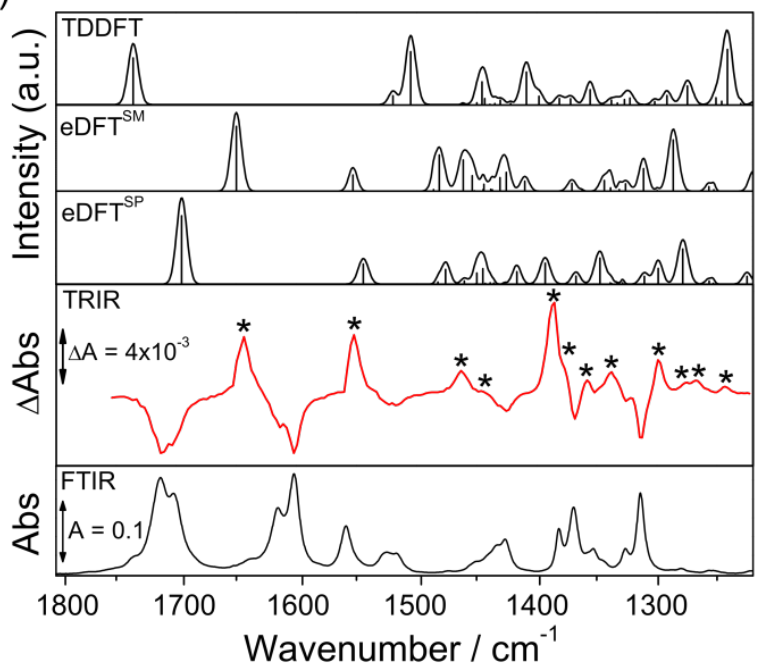

(c)




Figure 5. FTIR and TRIR spectra of (a) C151, (b) C102 and (c) C153 in acetonitrile- $d_{3} 100$ ps after excitation, compared to $\mathrm{S}_{1}$ frequency calculations: TDDFT, eDFT ${ }^{\mathrm{SM}}$ and $\mathrm{eDFT}^{\mathrm{SP}}$. Peaks labelled with $\mathrm{a} *$ are assigned to the $\mathrm{S}_{1}$ excited state.

Having examined both the TRIR spectra of the singlet excited state of C120b and compared to the calculated singlet IR spectra we have extended the analysis to other coumarins namely C151, C102 and C153 (Figure 5). For C151, a similar change was observed for the carbonyl mode following excitation at $266 \mathrm{~nm}$, with the carbonyl band downshifting from 1731 to $1666 \mathrm{~cm}^{-1}$ and another $\mathrm{S}_{1}$ excited state band observed at $1578 \mathrm{~cm}^{-1}$. By comparison with C120a, the carbonyl mode has a higher frequency in both the $S_{0}$ and $S_{1}$ states and the downshift following excitation is also reduced. Both of these observations follow from a reduction in the electron density at the carbonyl group on fluorination of the methyl substituent, and the changes in vibrational modes are also observed using DFT. The eDFT ${ }^{\mathrm{SM}}$ calculation significantly underestimates the gap between the carbonyl and the next highest frequency mode, with $\mathrm{eDFT}^{\mathrm{SP}}$ correctly predicting the frequency splitting while slightly overestimating the absolute values. Both eDFT predictions provide a good description of experiment compared with TDDFT, which overestimates the carbonyl band by the largest amount. Below $1550 \mathrm{~cm}^{-1}$, both eDFT predictions also match the $\mathrm{S}_{1}$ peaks better than TDDFT and both predict evenly spread out frequencies, which represent the experimental peaks well. In this part of the spectrum, TDDFT predicts a large region with no vibrational peaks between 1450 and $1325 \mathrm{~cm}^{-1}$, which is not observed in the experimental $\mathrm{S}_{1}$ spectrum.

The IR spectra of the two coumarins with ring-substituted 7-amino groups, C102 and C153, are shown in Figure 5. A similar increase in the frequencies of the carbonyl bands following fluorination (as in the case of $\mathrm{C} 120$ and C151) was observed, as well as a decrease in the downshift upon excitation (see Table 1). C102 and C153 both show highly congested TRIR spectra below $1500 \mathrm{~cm}^{-1}$ due to the additional degrees of freedom present following 
substitution at the 7-amino position. This makes comparison of the excited state predictions less straightforward in this region, and the analysis is therefore focused on the carbonyl and next highest frequency $S_{1}$ band, observed at 1643 and $1553 \mathrm{~cm}^{-1}$ for $\mathrm{C} 102$, and at 1680 and $1573 \mathrm{~cm}^{-1}$ for $\mathrm{C} 153$, respectively. For these two bands, $\mathrm{eDFT}^{\mathrm{SM}}$ provides a good match while $\mathrm{eDFT}^{\mathrm{SP}}$ still marginally overestimates the frequency of the carbonyl band. Similar to the other molecules, TDDFT overestimates the frequency for the carbonyl mode, especially for $\mathrm{C} 102$, where the overestimation is $93 \mathrm{~cm}^{-1}$ compared to only $52 \mathrm{~cm}^{-1}$ using eDFT ${ }^{\mathrm{SP}}$. The TDDFT spectrum of $\mathrm{C} 102$ also shows an underestimation of the peak at $1553 \mathrm{~cm}^{-1}$ in the TRIR by $c a .34 \mathrm{~cm}^{-1}$ and provides a significantly worse visual match when compared to the eDFT methods.

Table 1. Calculated and experimental frequencies $\left(\mathrm{cm}^{-1}\right)$ for the carbonyl modes of the 7-aminocoumarin states studied.

\begin{tabular}{lcccccc}
\hline Coumarin & $\mathrm{S}_{0}$ DFT & S $_{0}$ Exp. $^{b^{2}}$ & $\mathrm{~S}_{1}$ TDDFT & $\mathrm{S}_{1}$ eDFT $^{\mathrm{SM}}$ & $\mathrm{S}_{1}$ eDFT $^{\text {SP }}$ & $\mathrm{S}_{1}$ Exp. $^{b}$ \\
\hline $\mathrm{C} 120 \mathrm{a}$ & 1760 & 1716 & 1708 & 1613 & 1664 & 1632 \\
$\mathrm{C} 120 \mathrm{a}^{\mathrm{a}}$ & 1728 & 1716 & 1700 & 1612 & 1642 & 1632 \\
$\mathrm{C} 120 \mathrm{~b}$ & 1717 & 1684 & 1666 & 1610 & 1622 & 1591 \\
$\mathrm{C} 151$ & 1766 & 1731 & 1746 & 1638 & 1713 & 1666 \\
$\mathrm{C} 102$ & 1754 & 1713 & 1736 & 1650 & 1695 & 1643 \\
$\mathrm{C} 153$ & 1758 & 1730 & 1758 & 1673 & 1736 & 1680 \\
\hline
\end{tabular}

${ }^{a}$ Calculations include explicit acetonitrile- $d_{3}$ solvent.

${ }^{b}$ Experimental values obtained in acetonitrile- $d_{3}$.

\section{Conclusions}

TRIR spectra for the $\mathrm{S}_{1}$ state of a range of coumarin dyes have been reported and compared with calculated spectra from TDDFT and eDFT. For the molecules studied, the results demonstrate that eDFT gives accurate excited state IR spectra, providing an alternative approach to TDDFT. This is particularly the case for states that can be described by a single 
excitation from the ground state wave function. The results also show that using hybrid exchange-correlation functionals that are known to be accurate for ground state frequency calculations with standard scaling factors gives a good level of accuracy. For the functional used, the eDFT $\mathrm{S}_{1}$ spectra predicted for C120a and C151, show a significant improvement over the TDDFT predictions for both molecules. Further comparisons of the eDFT calculations show that the spin-purified and spin-mixed spectra give similar levels of agreement with experiment for the majority of modes tested, but with a slight improvement in both frequencies and visual match following spin-purification.

For $\mathrm{C} 102$ and $\mathrm{C} 153$, the carbonyl mode is a particularly important spectral feature and highlights the differences between the three methods. $\mathrm{eDFT}^{\mathrm{SP}}$ provides accuracy comparable to that of the ground state, $\mathrm{eDFT}^{\mathrm{SM}}$ predicts the carbonyl frequencies accurately but can suffer from artificial delocalization of the normal mode vector, whereas TDDFT tends to overestimate the carbonyl mode by up to $98 \mathrm{~cm}^{-1}$. The more accurate prediction of experimental frequencies and apparent better description of the carbonyl mode provided by eDFT, particularly following spin-purification, makes it especially useful for analysing and predicting the excited state spectra of the four coumarins studied. These results, combined with the increased computational speed of eDFT calculations compared to TDDFT calculations, indicate that eDFT has the potential to be a powerful tool for calculating singlet excited states, particularly for the prediction of excited state vibrational frequencies of large molecules/complex systems. ${ }^{68}$

\section{Acknowledgements}


We thank the University of Nottingham for funding, the STFC for access to the ULTRA facility, and the University of Nottingham for access to its High Performance Computing facility. MWG gratefully acknowledges receipt of a Royal Society Wolfson Merit Award. 


\section{References}

1. W. Koch and C. Holthausen, A Chemist's Guide to Density Functional Theory, Wiley-VCH, Weinheim, 2000.

2. M. W. D. Hanson-Heine, M. W. George and N. A. Besley, J. Phys. Chem. A, 2012, $116,4417-4425$.

3. C. Y. Lin, M. W. George and P. M. W. Gill, Aust. J. Chem., 2004, 57, 365-370.

4. M. W. D. Hanson-Heine, M. W. George and N. A. Besley, J. Chem. Phys., 2012, 136, 224102.

5. P. A. Summers, J. Dawson, F. Ghiotto, M. W. D. Hanson-Heine, K. Q. Vuong, E. S. Davies, X.-Z. Sun, N. A. Besley, J. McMaster, M. W. George and M. Schroeder, Inorg. Chem., 2014, 53, 4430-4439.

6. B. O. Roos, K. Andersson, M. P. Fulscher, P. A. Malmqvist, L. SerranoAndres, K. Pierloot and M. Merchan, Adv. Chem. Phys., 1996, 93, 219-331.

7. P. J. Knowles and H. J. Werner, Theor. Chim. Acta, 1992, 84, 95-103.

8. H. Koch and P. Jorgensen, J. Chem. Phys., 1990, 93, 3333-3344.

9. J. F. Stanton and R. J. Bartlett, J. Chem. Phys., 1993, 98, 7029-7039.

10. E. Runge and E. K. U. Gross, Phys. Rev. Lett., 1984, 52, 997-1000.

11. M. E. Casida, Recent advances in Density Functional Theory, Elsevier, Amsterdam, 1996.

12. F. Furche and R. Ahlrichs, J. Chem. Phys., 2002, 117, 7433-7447.

13. D. Rappoport and F. Furche, J. Chem. Phys., 2005, 122, 064105.

14. A. L. Sobolewski and W. Domcke, J. Phys. Chem. A, 2004, 108, 10917-10922.

15. Y. Liu, J. Ding, R. Liu, D. Shi and J. Sun, J. Comput. Chem., 2009, 30, 2723-2727.

16. Y.-F. Liu, D.-P. Yang, D.-H. Shi and J.-F. Sun, J. Comput. Chem., 2011, 32, 34753484. 
17. W. Zhang, Z. Lan, Z. Sun and K. J. Gaffney, J. Phys. Chem. B, 2012, 116, 1152711536.

18. K. Bartl, A. Funk and M. Gerhards, ChemPhysChem, 2009, 10, 1882-1886.

19. L. D. Movsisyan, M. D. Peeks, G. M. Greetham, M. Towrie, A. L. Thompson, A. W. Parker and H. L. Anderson, J. Am. Chem. Soc., 2014, 136, 17996-18008.

20. A. Dreuw and M. Head-Gordon, J. Am. Chem. Soc., 2004, 126, 4007-4016.

21. A. Dreuw, J. L. Weisman and M. Head-Gordon, J. Chem. Phys., 2003, 119, 29432946.

22. M. E. Casida, C. Jamorski, K. C. Casida and D. R. Salahub, J. Chem. Phys., 1998, $108,4439-4449$.

23. D. J. Tozer and N. C. Handy, J. Chem. Phys., 1998, 109, 10180-10189.

24. N. A. Besley, M. J. G. Peach and D. J. Tozer, Phys. Chem. Chem. Phys., 2009, 11, 10350-10358.

25. T. Yanai, D. P. Tew and N. C. Handy, Chem. Phys. Lett., 2004, 393, 51-57.

26. R. M. Edkins, A. Wriglesworth, K. Fucke, S. L. Bettington and A. Beeby, Dalton Trans., 2011, 40, 9672-9678.

27. F. L. Thorp-Greenwood, J. A. Platts and M. P. Coogan, Polyhedron, 2014, 67, 505512.

28. M. J. G. Peach, T. Helgaker, P. Salek, T. W. Keal, O. B. Lutnaes, D. J. Tozer and N. C. Handy, Phys. Chem. Chem. Phys., 2006, 8, 558-562.

29. J. Plotner, D. J. Tozer and A. Dreuw, J. Chem. Theory Comput., 2010, 6, 2315-2324.

30. M. W. D. Hanson-Heine, M. W. George and N. A. Besley, J. Chem. Phys., 2013, 138, 064101.

31. C. L. Cheng, Q. Wu and T. Van Voorhis, J. Chem. Phys., 2008, 129, 124112.

32. P. S. Bagus, Phys. Rev., 1965, 139, A619-A634. 
33. H. L. Hsu, E. R. Davidson and R. M. Pitzer, J. Chem. Phys., 1976, 65, 609-613.

34. A. N. Debrito, N. Correia, S. Svensson and H. Agren, J. Chem. Phys., 1991, 95, 29652974.

35. A. T. B. Gilbert, N. A. Besley and P. M. W. Gill, J. Phys. Chem. A, 2008, 112, 1316413171.

36. A. J. W. Thom and M. Head-Gordon, Phys. Rev. Lett., 2008, 101, 193001.

37. T. Baruah, M. Olguin and R. R. Zope, J. Chem. Phys., 2012, 137, 084316.

38. J. A. Calladine, R. Horvath, A. J. Davies, A. Wriglesworth, X.-Z. Sun and M. W. George, Appl. Spectrosc., In Press.

39. A. L. Pitts, A. Wriglesworth, X.-Z. Sun, J. A. Calladine, S. D. Zaric, M. W. George and M. B. Hall, J. Am. Chem. Soc., 2014, 136, 8614-8625.

40. V. K. Sharma, P. D. Saharo, N. Sharma, R. C. Rastogi, S. K. Ghoshal and D. Mohan, Spectrochim. Acta Mol. Biomol. Spectros., 2003, 59, 1161-1170.

41. A. Pedone, J. Bloino and V. Barone, J. Phys. Chem. C, 2012, 116, 17807-17818.

42. R. Sanchez-de-Armas, M. Angel San Miguel, J. Oviedo and J. Fdez Sanz, Phys. Chem. Chem. Phys., 2012, 14, 225-233.

43. R. Improta, V. Barone, G. Scalmani and M. J. Frisch, J. Chem. Phys., 2006, 125.

44. R. J. Cave, K. Burke and E. W. Castner, J. Phys. Chem. A, 2002, 106, 9294-9305.

45. W. Zhao, Y. Ding and Q. Xia, J. Comput. Chem., 2011, 32, 545-553.

46. H. Pal, S. Nad and M. Kumbhakar, J. Chem. Phys., 2003, 119, 443-452.

47. S. Nad and H. Pal, J. Phys. Chem. A, 2001, 105, 1097-1106.

48. G. Jones, W. R. Jackson, C. Choi and W. R. Bergmark, J. Phys. Chem., 1985, 89, 294-300.

49. A. P. Scott and L. Radom, J. Phys. Chem., 1996, 100, 16502-16513.

50. J. P. Merrick, D. Moran and L. Radom, J. Phys. Chem. A, 2007, 111, 11683-11700. 
51. A. D. Boese, W. Klopper and J. M. L. Martin, Int. J. Quant. Chem., 2005, 104, 830845.

52. C. Adamo and V. Barone, J. Chem. Phys., 1999, 110, 6158-6170.

53. M. Biczysko, P. Panek, G. Scalmani, J. Bloino and V. Barone, J. Chem. Theory Comput., 2010, 6, 2115-2125.

54. K. Rechthaler and G. Kohler, Chem. Phys., 1994, 189, 99-116.

55. S. Nad, M. Kumbhakar and H. Pal, J. Phys. Chem. A, 2003, 107, 4808-4816.

56. T. L. Arbeloa, F. L. Arbeloa, M. J. Tapia and I. L. Arbeloa, J. Phys. Chem., 1993, 97, 4704-4707.

57. G. Jones, W. R. Jackson, S. Kanoktanaporn and A. M. Halpern, Opt. Commun., 1980, $33,315-320$.

58. A. Pedone, E. Gambuzzi, V. Barone, S. Bonacchi, D. Genovese, E. Rampazzo, L. Prodi and M. Montalti, Phys. Chem. Chem. Phys., 2013, 15, 12360-12372.

59. See supplementary material at [URL will be inserted by AIP] for vertical excitation energies, geometry coordinates, ground state spectra, and additional syntheic details.

60. G. M. Greetham, P. Burgos, Q. Cao, I. P. Clark, P. S. Codd, R. C. Farrow, M. W. George, M. Kogimtzis, P. Matousek, A. W. Parker, M. R. Pollard, D. A. Robinson, Z.-J. Xin and M. Towrie, Appl. Spectrosc., 2010, 64, 1311-1319.

61. Y. Shao, L. F. Molnar, Y. Jung, J. Kussmann, C. Ochsenfeld, S. T. Brown, A. T. B. Gilbert, L. V. Slipchenko, S. V. Levchenko, D. P. O'Neill, R. A. DiStasio, Jr., R. C. Lochan, T. Wang, G. J. O. Beran, N. A. Besley, J. M. Herbert, C. Y. Lin, T. Van Voorhis, S. H. Chien, A. Sodt, R. P. Steele, V. A. Rassolov, P. E. Maslen, P. P. Korambath, R. D. Adamson, B. Austin, J. Baker, E. F. C. Byrd, H. Dachsel, R. J. Doerksen, A. Dreuw, B. D. Dunietz, A. D. Dutoi, T. R. Furlani, S. R. Gwaltney, A. Heyden, S. Hirata, C.-P. Hsu, G. Kedziora, R. Z. Khalliulin, P. Klunzinger, A. M. 
Lee, M. S. Lee, W. Liang, I. Lotan, N. Nair, B. Peters, E. I. Proynov, P. A. Pieniazek, Y. M. Rhee, J. Ritchie, E. Rosta, C. D. Sherrill, A. C. Simmonett, J. E. Subotnik, H. L. Woodcock, III, W. Zhang, A. T. Bell, A. K. Chakraborty, D. M. Chipman, F. J. Keil, A. Warshel, W. J. Hehre, H. F. Schaefer, III, J. Kong, A. I. Krylov, P. M. W. Gill and M. Head-Gordon, Phys. Chem. Chem. Phys., 2006, 8, 3172-3191.

62. A. D. Becke, J. Chem. Phys., 1993, 98, 5648-5652.

63. P. J. Stephens, F. J. Devlin, C. F. Chabalowski and M. J. Frisch, J. Phys. Chem., 1994, 98, 11623-11627.

64. P. M. W. Gill, B. G. Johnson and J. A. Pople, Chem. Phys. Lett., 1993, 209, 506-512.

65. M. P. Andersson and P. Uvdal, J. Phys. Chem. A, 2005, 109, 2937-2941.

66. T. Ziegler, A. Rauk and E. J. Baerends, Theor. Chim. Acta, 1977, 43, 261-271.

67. T. Kowalczyk, S. R. Yost and T. Van Voorhis, J. Chem. Phys., 2011, 134, 054128.

68. J. Liu and W. Liang, J. Chem. Phys., 2011, 135, 014113. 